\title{
Dynamics of Non-viscously Damped Distributed Parameter Systems
}

\author{
Sondipon Adhikari ${ }^{*}$ \\ University of Bristol, Bristol, United Kingdom \\ Yongjun Lei ${ }^{\dagger}$ \\ National University of Defense Technology, Changsha, PR China \\ Michael I. Friswell: \\ University of Bristol, Bristol, United Kingdom
}

\begin{abstract}
Linear dynamics of Euler-Bernoulli beams with non-viscous non-local damping is considered. It is assumed that the damping force at a given point in the beam depends on the past history of velocities at different points via convolution integrals over exponentially decaying kernel functions. Conventional viscous and viscoelastic damping models can be obtained as special cases of this general damping model. The equation of motion of the beam with such general damping model results in a linear partial integro-differential equation. Exact closed-form expressions of the natural frequencies and mode-shapes of the beam are derived. The analytical method is capable of handling complex boundary conditions. Numerical examples are provided to illustrate the new results.
\end{abstract}

\section{Introduction}

$\mathrm{T}$ HE finite element method, coupled with model updating techniques, allow us to accurately model the 1 mass and the stiffness properties of complex engineering structures and subsequently to analyze their dynamics. Determination of the dynamic response, which is crucial for the design of a structure, not only depends on the mass and stiffness properties but also heavily depends on the energy dissipation properties or 'damping'. The capabilities of modern design tools, in terms of the modelling and analyzing of damping properties, are not as advanced as for the mass and stiffness properties. There are several reasons behind this: (a) by contrast with inertia and stiffness forces, it is not in general clear which state variables are relevant to determine the damping forces, (b) the spatial location of the damping sources are generally unclear - often the structural joints are more responsible for the energy dissipation than the (solid) material, (c) the functional form of the damping model is difficult to establish experimentally, and finally, (d) even if one manages to address the previous issues, what parameters should be used in a chosen model is still very much an open problem. Because of these difficulties modelling of damping from first principles is very difficult, if not impossible, for complex engineering structures. Over the years, and even now, structural dynamicists have bypassed these problems by using the viscous damping model. A vast literature, starting from Lord Rayleigh's ${ }^{1}$ classic monograph, 'Theory of Sound', is available on the viscous damping model. With a viscous damping model, it is assumed that the instantaneous generalized velocities are the only relevant state variables that determine damping. Viscous damping is by no means the only damping model

\footnotetext{
${ }^{*}$ Lecturer, Department of Aerospace Engineering University of Bristol Queens Building, University Walk, Bristol BS8 1TR, UK, AIAA Member.

${ }^{\dagger}$ Associate Professor, College of Aerospace and Material Engineering, National University of Defense Technology, Changsha 410073, PR China

${ }^{\ddagger}$ Professor, Department of Aerospace Engineering University of Bristol Queens Building, University Walk, Bristol BS8 $1 T R$, UK, AIAA Member.
} 
within the scope of linear analysis. Any causal model which makes the energy dissipation functional nonnegative is a possible candidate for a damping model. Non-viscous damping models in general have more parameters and therefore are more likely to have a better match with experimental measurements.

A key issue in considering non-viscous damping is to decide on an appropriate damping model. There have been detailed studies of material damping and damping of specific structural components. Lazan, ${ }^{2}$ Bert ${ }^{3}$ and Ungar $^{4}$ have given excellent accounts of different mathematical methods for modelling damping in a (solid) material and their engineering applications. The book by Nashif et al. ${ }^{5}$ presents more recent studies in this area. In the context of linear MDOF discrete systems, Adhikari ${ }^{6}$ and Wagner and Adhikari ${ }^{7}$ considered a nonviscous damping model in which the damping forces are assumed to depend on velocity time histories as well as instantaneous velocities. In the context of distributed parameter systems, Banks and Inman ${ }^{8}$ considered non-viscous damping models in Euler-Bernoulli beams. They considered four different models of damping: viscous air damping, Kelvin-Voigt damping, time hysteresis damping and spatial hysteresis damping, and used a spline inverse procedure to form a least-square fit to the experimental data. It was observed that the spatial hysteresis model combined with a viscous air damping model gave the best quantitative agreement with the experimental time histories. This study motivates us to consider general damping models in distributed parameter systems. The aim of this paper is to extend conventional modal analysis to an EulerBernoulli beam with linear general damping.

\section{Linear Damping Models for Distributed Parameter System}

A linear damped continuous dynamic system in which the displacement variable $u(\mathbf{r}, t)$, where $\mathbf{r}$ is the spatial position vector and $t$ is time, specified in some domain $\mathcal{D}$, is governed by a linear partial differential equation

$$
\rho(\mathbf{r}) \ddot{u}(\mathbf{r}, t)+\mathcal{L}_{1} \dot{u}(\mathbf{r}, t)+\mathcal{L}_{2} u(\mathbf{r}, t)=p(\mathbf{r}, t) ; \quad \mathbf{r} \in \mathcal{D}, \quad t \in[0, T]
$$

with homogeneous linear boundary conditions of the form

$$
\mathcal{M}_{1} u(\mathbf{r}, t)=0 ; \quad \mathbf{r} \in \Gamma_{1} \quad \text { and } \quad \mathcal{M}_{2} \dot{u}(\mathbf{r}, t)=0 ; \quad \mathbf{r} \in \Gamma_{2}
$$

specified on some boundary surfaces $\Gamma_{1}$ and $\Gamma_{2}$. In the above equation $\rho(\mathbf{r})$ is the mass distribution of the system, $p(\mathbf{r}, t)$ is the distributed time-varying forcing function, $\mathcal{L}_{2}$ is the spatial self-adjoint stiffness operator and $\mathcal{M}_{1}$ and $\mathcal{M}_{2}$ are linear operators acting on the boundary. The damping operator $\mathcal{L}_{1}$ can be written in the form

$$
\mathcal{L}_{1} \dot{u}(\mathbf{r}, t)=\int_{\mathcal{D}} \int_{-\infty}^{t} C_{1}(\mathbf{r}, \boldsymbol{\xi}, t-\tau) \dot{u}(\boldsymbol{\xi}, \tau) d \tau d \boldsymbol{\xi}
$$

where $C_{1}(\mathbf{r}, \boldsymbol{\xi}, t)$ is the kernel function. The velocities $\dot{u}(\boldsymbol{\xi}, \tau)$ at different time instants and spatial locations are coupled through this kernel function. Kernel functions that serve similar purposes have been described by different names in different subjects (for example, retardation functions, heredity functions, after-effect functions, relaxation functions), and different models have been used to describe them. In principle, any function that makes the rate of energy dissipation,

$$
\mathcal{F}(t)=\frac{1}{2} \int_{\mathcal{D}}\left\{\int_{\mathcal{D}} \int_{-\infty}^{t} C_{1}(\mathbf{r}, \boldsymbol{\xi}, t-\tau) \dot{u}(\boldsymbol{\xi}, \tau) d \tau d \boldsymbol{\xi}\right\} \dot{u}(\mathbf{r}, t) d \mathbf{r}
$$

non-negative can be used as a kernel function. Equation (1) together with Eq. (3) represents a continuous dynamic system with general linear damping. It may be noted that if $\mathcal{L}_{1}=0$ in Eq. (1), i.e. an undamped system, or if the system satisfies the criteria given by Caughey and O'Kelly, ${ }^{9}$ then the system will possess classical normal modes. However, due to the general nature of the operator $\mathcal{L}_{1}$ as described by Eq. (3), there is no definite reason why the system should have classical normal modes. Thus the mode shapes and natural frequencies of such systems in general will be complex in nature. In this context we wish to note that the system expressed by (1) and the damping operator defined in (3) represents a partial integro-differential equation with the boundary conditions given in Eq. (2). In this paper we are interested in the natural frequencies and mode shapes of the system. Exact closed-form expressions of such quantities for the general case are difficult to obtain. We make the following general assumptions:

- the mass and stiffness distributions are homogeneous, that is, they do not vary with the position vector $\mathbf{r}$, and 
- the damping kernel function $C_{1}(\mathbf{r}, \boldsymbol{\xi}, t)$ is separable in space and time.

Next we discuss some special cases and explain their physical meaning.

We limit our discussions to the case of homogenous mass and stiffness distributions. We begin by considering special cases of the damping kernel function $C_{1}(\mathbf{r}, \boldsymbol{\xi}, t)$, starting from the simple viscous model to the more general non-viscous model. In general it is assumed that the kernel function is separable in space and time.

\section{A. Non-proportional Viscous Damping}

Initially, we consider a kernel function given by a delta function in both space and time, that is

$$
C_{1}(\mathbf{r}, \boldsymbol{\xi}, t-\tau)=C(\mathbf{r}) \delta(\mathbf{r}-\boldsymbol{\xi}) \delta(t-\tau) .
$$

This spatial delta function physically means that the damping force is 'locally reacting' and the time delta function implies that the force depends only on the instantaneous value of the motion. From Eq. (4) the rate of energy dissipation leads to the familiar Rayleigh dissipation function defined by

$$
\mathcal{F}(t)=\frac{1}{2} \int_{\mathcal{D}} C(\mathbf{r}) \dot{u}^{2}(\mathbf{r}, t) d \mathbf{r}
$$

This model represents the well known 'viscous damping' model, however, no assumption about proportional damping has been made in this study.

\section{B. Viscoelastic Damping (Time Hysteresis)}

Here we consider that the kernel function is a delta function in space but depends on the past time histories via a convolution integral as described by Eq. (3). The kernel takes form

$$
C_{1}(\mathbf{r}, \boldsymbol{\xi}, t-\tau)=C(\mathbf{r}) g(t-\tau) \delta(\mathbf{r}-\boldsymbol{\xi}) .
$$

This represents a locally reacting viscoelastic damping model. Such damping models are also called 'time hysteresis' damping. 8 Physically this kind of model represents a damping force that depends on the past time history through a convolution integral over the kernel function.

\section{Non-local Viscous Damping (Spatial Hysteresis)}

In this case the kernel function is assumed to be a delta function in time but depends on the spatial distribution of the velocities via a convolution integral as described by Eq. (3). The kernel function in this case is given by

$$
C_{1}(\mathbf{r}, \boldsymbol{\xi}, t-\tau)=C(\mathbf{r}) c(\mathbf{r}-\boldsymbol{\xi}) \delta(t-\tau)
$$

This kind of damping is expected in foam material. Physically this model implies that velocities at different points can affect the damping force at a given point.

\section{Non-local Viscoelastic Damping}

This is the most general form of damping we consider in our study. The only assumption here is that the kernel function is separable in space and time so the kernel takes form of

$$
C_{1}(\mathbf{r}, \boldsymbol{\xi}, t-\tau)=C(\mathbf{r}) c(\mathbf{r}-\boldsymbol{\xi}) g(t-\tau)
$$

Physically this model represents non-local viscoelastic damping, for example, in a beam with viscoelastic material or an actively controlled piezoelectric patch. All the previous three damping models can be identified as special cases of this model. 


\section{E. Parametrization of the General Damping Model}

The damping model proposed here is perhaps the most general linear damping model considered in the literature. By solving Eq. (1), together with boundary the conditions in (2) it is possible to derive the expressions for natural frequencies, mode shapes and dynamic response. The aim of this study is to understand the nature of the natural frequencies and the mode shapes by comparing the results arising from the four special cases introduced above. Once these quantities are well understood, it may be possible to recognize a similar pattern from experiments which would indicate the form of damping model present. Once a damping model is selected, it would then be possible to fit its parameters. Most current methods assume a viscous model a priori and therefore rule out the possibility of identifying any other damping mechanism that might be present in a structure. We hope that the general model proposed here will give a more realistic picture of the damping mechanisms. In order to proceed further, it is necessary to use some kind of plausible functional form of the kernel functions in space and time. We start with energy considerations associated with the general case in Eq. (9).

Substituting the expression of the kernel function from Eq. (9) into Eq. (4) and taking the Fourier transform we have

$$
\hat{\mathcal{F}}(\omega)=\frac{\omega^{2}}{2} \int_{\mathcal{D}} \int_{\mathcal{D}} U^{*}(\boldsymbol{\xi}, \omega) C(\mathbf{r}) c(\mathbf{r}-\boldsymbol{\xi}) G(\omega) U(\mathbf{r}, \omega) d \boldsymbol{\xi} d \mathbf{r}
$$

Here $(\bullet)^{*}$ denotes complex conjugation, and $G(\omega)$ and $U(\bullet, \omega)$ are the Fourier transforms of $g(t)$ and $u(\bullet, t)$ respectively. For a physically realistic model of damping, the real part of the dissipation energy should be non-negative at all frequencies, i.e., $\Re[\hat{\mathcal{F}}(\omega)] \geq 0, \forall \omega$, where $\Re(\bullet)$ represents the real part. This gives a restriction on the kernel functions and we have following general result:

Condition: For a physically realistic model of damping $\Re[\hat{\mathcal{F}}(\omega)] \geq 0, \forall \omega$ or

$$
\Re\left[G(\omega) \int_{\mathcal{D}} \int_{\mathcal{D}} C(\mathbf{r}) c(\mathbf{r}-\boldsymbol{\xi}) U^{*}(\boldsymbol{\xi}, \omega) U(\mathbf{r}, \omega) d \boldsymbol{\xi} d \mathbf{r}\right] \geq 0, \forall \omega .
$$

This can be seen from Eq. (10) by noticing that for a real value of driving frequency $\omega^{2} \geq 0$.

There can be several models which would satisfy the condition in Eq. (11). For example, the viscous model in (5) satisfies this condition for positive $C(\mathbf{r})$. The non-viscous model in Eq. (7) also satisfies this condition if $C(\mathbf{r}) \Re[G(\omega)]$ is positive. In this study we choose the following functional forms:

$$
\begin{aligned}
g(t) & =g_{\infty} \mu \exp (-\mu t) \quad \text { so that } \quad G(\omega)=\frac{g_{\infty} \mu}{\mathrm{i} \omega+\mu} \\
\text { and } \quad c(\mathbf{r}-\boldsymbol{\xi}) & =\frac{\alpha}{2} \exp (-\alpha|\mathbf{r}-\boldsymbol{\xi}|), \quad C(\mathbf{r}) \geq 0
\end{aligned}
$$

It can be shown that condition (11) will always be satisfied when $g_{\infty}, \mu$ and $\alpha$ are all positive constants. If $\alpha \rightarrow \infty, \mu \rightarrow \infty$ one obtains the standard viscous model in (5), if $\alpha \rightarrow \infty$ and $\mu$ is finite one obtains the non-viscous model in (7) and if $\alpha$ is finite but $\mu \rightarrow \infty$ one obtains the damping model in (8). Thus, it is expected that the natural frequencies and the mode shapes in these parameter cases of the general model (9) should approach the appropriate special cases.

In this paper we consider modal analysis of an Euler-Bernoulli beam with general linear damping given by equation (9). The analytical approach proposed is based on transfer function method. ${ }^{10}$ In the next sections closed-form expressions of natural frequencies and mode shapes are derived for different boundary conditions.

\section{Governing Equation of Motion}

The Euler-Bernoulli beam considered in this study is shown in figure 1. The left and right coordinate of the beam are denoted by $x_{L}$ and $x_{R}$ respectively. The beam has a non-local viscoelastic damping patch between $x_{1}$ and $x_{2}$. It is assumed that the elastic properties of the beam are uniformly distributed with bending rigidity $E I$ and mass density $\rho A$. The equation of motion of the part with the damping patch can 


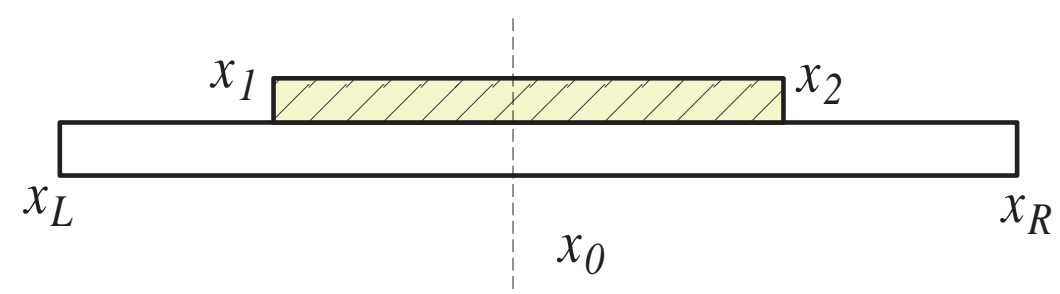

Figure 1. Euler-Bernoulli beam with non-viscous damping patch.

be expressed by

$$
\begin{aligned}
& E I \frac{\partial^{4} w(x, t)}{\partial x^{4}}+ \rho A \frac{\partial^{2} w(x, t)}{\partial t^{2}}+\int_{x_{1}}^{x_{2}} \int_{-\infty}^{t} \frac{\alpha}{2} \exp (-\alpha|x-\xi|) \\
&\left.g_{\infty} \mu \exp (-\mu(t-\tau)) \frac{\partial w(\xi, t)}{\partial t}\right|_{t=\tau} d \xi d \tau=f(x, t) \quad \text { when } \quad x \in\left[x_{1}, x_{2}\right]
\end{aligned}
$$

The equation of motion of the part outside the damping patch can be expressed by

$$
E I \frac{\partial^{4} w(x, t)}{\partial x^{4}}+\rho A \frac{\partial^{2} w(x, t)}{\partial t^{2}}+C_{0} \frac{\partial w(x, t)}{\partial t}=f(x, t) \quad \text { when } \quad x \in\left(x_{L}, x_{1}\right) \cup\left(x_{2}, x_{R}\right)
$$

Appropriate boundary conditions must be satisfied at $x=x_{L}$ and at $x=x_{R}$. In addition to this we need to satisfy relevant continuity conditions at the internal points $x_{1}$ and $x_{2}$. Discussions on different boundary conditions will be given in section VII. In what follows, Eqs. (14) and (15) are solved separately and the solutions are combined to obtain the overall solution. We begin with the solution of Eq. (14).

\section{Transfer Matrix Solution for the Section with Non-local Viscoelastic Damping Patch}

Assuming zero initial conditions, the Laplace transform of Eq. (14) leads to

$$
E I W^{I V}(x, s)+s^{2} \rho A W(x, s)+\int_{x_{1}}^{x_{2}} \frac{\alpha}{2} \exp (-\alpha|x-\xi|) \frac{s g_{\infty} \mu}{\mu+s} W(\xi, s) d \xi=F(x, s)
$$

$$
x \in\left(x_{1}, x_{2}\right)
$$

Here $s$ is the complex Laplace parameter, $W(x, s)$ and $F(x, s)$ are the Laplace transforms of $w(x, t)$ and $f(x, t)$ respectively. The roman superscripts, for example $(\bullet)^{I V}$, denote the order of derivative with respect to the spatial variable $x$. It is useful to separate the contribution arising from the term $|x-\xi|$ in Eq. (16) as

$$
\begin{aligned}
E I W^{I V}(x, s)+s^{2} \rho A W(x, s) & +\int_{x_{1}}^{x} s G(s) \exp (-\alpha(x-\xi)) W(\xi, s) d \xi \\
& +\int_{x}^{x_{2}} s G(s) \exp (\alpha(x-\xi)) W(\xi, s) d \xi=F(x, s) \\
\text { where } \quad G(s)=\frac{\alpha}{2} \frac{g_{\infty} \mu}{\mu+s} &
\end{aligned}
$$

Differentiating Eq. (17) with respect to the spatial variable $x$ twice one obtains

$$
\begin{aligned}
E I W^{V}(x, s)+s^{2} \rho A W^{I}(x, s) & -\alpha s G(s) \int_{x_{1}}^{x} \exp (-\alpha(x-\xi)) W(\xi, s) d \xi \\
& +\alpha s G(s) \int_{x}^{x_{2}} \exp (\alpha(x-\xi)) W(\xi, s) d \xi=F^{I}(x, s)
\end{aligned}
$$

$$
5 \text { of } 16
$$


and

$$
\begin{aligned}
& E I W^{V I}(x, s)+s^{2} \rho A W^{I I}(x, s)+\alpha^{2} s G(s) \int_{x_{1}}^{x} \exp (-\alpha(x-\xi)) W(\xi, s) d \xi \\
& +\alpha^{2} s G(s) \int_{x}^{x_{2}} \exp (\alpha(x-\xi)) W(\xi, s) d \xi-2 \alpha G(s) W(x, s)=F^{I I}(x, s)
\end{aligned}
$$

Using Eqs. (17) and (20) we have

$$
\begin{aligned}
E I W^{V I}(x, s)+s^{2} \rho A W^{I I}(x, s) & -\alpha^{2}\left[E I W^{I V}(x, s)+s^{2} \rho A W(x, s)\right] \\
& -2 \alpha G(s) W(x, s)=F^{I I}(x, s)-\alpha^{2} F(x, s)
\end{aligned}
$$

Equation (21) is a sixth order ordinary differential equation. This equation can be solved by transforming into first-order form. Recall that the solution of a sixth order ordinary differential equation requires six boundary conditions corresponding to $W(x, s), \cdots, W^{V I}(x, s)$. However, the physics of the problem gives only four boundary conditions. In what follows, the 'unknown' boundary conditions corresponding to the higher order derivatives of $W(x, s)$ are expressed as a linear combination of the lower order derivatives.

We define the state vector $\boldsymbol{\eta}(x, s)$ and partition it as

$$
\boldsymbol{\eta}(x, s)=\left\{\begin{array}{l}
\mathbf{u}(x, s) \\
\mathbf{v}(x, s)
\end{array}\right\} \in \mathbb{C}^{6}
$$

where

$$
\begin{aligned}
\mathbf{u}(x, s) & =\left\{W(x, s), W^{I}(x, s), W^{I I}(x, s), W^{I I I}(x, s)\right\}^{T} \in \mathbb{C}^{4} \\
\text { and } \quad \mathbf{v}(x, s) & =\left\{W^{I V}(x, s), W^{V}(x, s)\right\}^{T} \in \mathbb{C}^{2}
\end{aligned}
$$

Using the state vector in Eq. (22), Eq. (21) can be cast in matrix form as

$$
\frac{d}{d x} \boldsymbol{\eta}(x, s)=\boldsymbol{\Phi}(s) \boldsymbol{\eta}(x, s)+\mathbf{q}(x, s), \quad x \in\left(x_{1}, x_{2}\right)
$$

where

$$
\boldsymbol{\Phi}(s)=\left[\begin{array}{cccccc}
0 & 1 & 0 & 0 & 0 & 0 \\
0 & 0 & 1 & 0 & 0 & 0 \\
0 & 0 & 0 & 1 & 0 & 0 \\
0 & 0 & 0 & 0 & 1 & 0 \\
0 & 0 & 0 & 0 & 0 & 1 \\
\frac{2 \alpha s G+\alpha^{2} s^{2} \rho A}{E I} & 0 & -\frac{s^{2} \rho A}{E I} & 0 & \alpha^{2} & 0
\end{array}\right], \mathbf{q}(x, s)=\left\{\begin{array}{c}
0 \\
0 \\
0 \\
0 \\
0 \\
\frac{F^{I I}(x, s)-\alpha^{2} F(x, s)}{E I}
\end{array}\right\}
$$

The solution of Eq. (25) can be expressed as

$$
\boldsymbol{\eta}(x, s)=\int_{x_{0}}^{x} \exp (\mathbf{\Phi}(s)(x-\xi)) \mathbf{q}(\xi, s) d \xi+\exp \left(\mathbf{\Phi}(s)\left(x-x_{0}\right)\right) \boldsymbol{\eta}_{0}(s), \quad x \in\left(x_{1}, x_{2}\right)
$$

where $\boldsymbol{\eta}_{0}(s)=\boldsymbol{\eta}\left(x_{0}, s\right)=\left\{\boldsymbol{\eta}_{0 i}(s)\right\},(i=1,2, \ldots, 6)$ is an undetermined vector. The point $x_{0}$ can be any point between $x_{1}$ and $x_{2}$. The displacement $W(x, s)$ can be obtained from $\boldsymbol{\eta}(x, s)$ as

$$
W(x, s)=\boldsymbol{\eta}_{1}(x, s)
$$

where $\boldsymbol{\eta}_{1}(x, s)$ is the first element of $\boldsymbol{\eta}(x, s)$. For simplification we define the auxiliary quantities

$$
\begin{aligned}
\boldsymbol{\eta}_{q}(x, s) & =\int_{x_{0}}^{x} \exp (\mathbf{\Phi}(s)(x-\xi)) \mathbf{q}(\xi, s) d \xi=\left\{\boldsymbol{\eta}_{q_{i}}(x, s)\right\}, \quad i=1,2, \cdots, 6 \\
\mathbf{\Psi}(x, s) & =\exp \left(\mathbf{\Phi}(s)\left(x-x_{0}\right)\right)=\left[\boldsymbol{\Psi}_{i j}(x, s)\right], \quad i, j=1,2, \cdots, 6
\end{aligned}
$$


Using the definition of state vectors of $\mathbf{u}(x, s), \mathbf{v}(x, s)$, and $\boldsymbol{\eta}(x, s)$, Eq. (28) can be rewritten as:

$$
\begin{aligned}
W(x, s) & =\boldsymbol{\eta}_{q 1}(x, s)+\sum_{i=1}^{6} \boldsymbol{\Psi}_{1 i}(x, s) \boldsymbol{\eta}_{0 i}(s) \\
& =\boldsymbol{\eta}_{q 1}(x, s)+\sum_{i=1}^{4} \boldsymbol{\Psi}_{1 i}(x, s) \mathbf{u}_{0 i}(s)+\sum_{i=5}^{6} \boldsymbol{\Psi}_{1(i)}(x, s) \mathbf{v}_{0(i-4)}(s)
\end{aligned}
$$

where $\boldsymbol{\eta}_{q 1}(x, s)$ is the first element of $\boldsymbol{\eta}_{q}(x, s)$ and $\mathbf{u}_{0}(s)=\mathbf{u}\left(x_{0}, s\right)$ and $\mathbf{v}_{0}(s)=\mathbf{v}\left(x_{0}, s\right)$ respectively. Substituting $x=x_{0}$ in Eqs. (17) and (19) one has

$$
\begin{aligned}
E I W^{I V}\left(x_{0}, s\right)+s^{2} \rho A W\left(x_{0}, s\right)+s G(s)\left[\int_{x_{1}}^{x_{0}} \exp \left(\alpha\left(\xi-x_{0}\right)\right) W(\xi, s) d \xi\right. & \\
& \left.+\int_{x_{0}}^{x_{2}} \exp \left(\alpha\left(x_{0}-\xi\right)\right) W(\xi, s) d \xi\right]=F\left(x_{0}, s\right) \\
E I W^{V}\left(x_{0}, s\right)+s^{2} \rho A W^{I}\left(x_{0}, s\right)-\alpha s G(s)\left[\int_{x_{1}}^{x_{0}} \exp \left(\alpha\left(\xi-x_{0}\right)\right) W(\xi, s) d \xi d \tau\right. & \left.-\int_{x_{0}}^{x_{2}} \exp \left(\alpha\left(x_{0}-\xi\right)\right) W(\xi, s) d \xi\right]=F^{I}\left(x_{0}, s\right)
\end{aligned}
$$

Substituting $W(x, s)$ from Eq. (31) into Eqs. (32) and (33) and performing the integration we have

$$
\begin{aligned}
& E I W^{I V}\left(x_{0}, s\right)+s^{2} \rho A W\left(x_{0}, s\right)+s G(s)\left[\sum_{i=1}^{4} \boldsymbol{\Theta}_{1 i}(s) \mathbf{u}_{0 i}(s)+\sum_{i=5}^{6} \boldsymbol{\Theta}_{1 i}(s) \mathbf{v}_{0(i-4)}(s)\right]=\bar{F}\left(x_{0}, s\right) \\
& E I W^{V}\left(x_{0}, s\right)+s^{2} \rho A W^{I}(0, s)+\alpha s G(s)\left[\sum_{i=1}^{4} \boldsymbol{\Xi}_{1 i}(s) \mathbf{u}_{0 i}(s)+\sum_{i=5}^{6} \boldsymbol{\Xi}_{1 i}(s) \mathbf{v}_{0(i-4)}(s)\right]=\bar{F}^{I}\left(x_{0}, s\right)
\end{aligned}
$$

where

$$
\begin{gathered}
\boldsymbol{\Theta}_{1 i}=\int_{x_{1}}^{x_{0}} \exp \left(\alpha\left(\xi-x_{0}\right)\right) \boldsymbol{\Psi}_{1 i}(\xi, s) d \xi+\int_{x_{0}}^{x_{2}} \exp \left(\alpha\left(x_{0}-\xi\right)\right) \boldsymbol{\Psi}_{1 i}(\xi, s) d \xi, \quad i=1, \cdots, 6 \\
\boldsymbol{\Xi}_{1 i}=-\int_{x_{1}}^{x_{0}} \exp \left(\alpha\left(\xi-x_{0}\right)\right) \boldsymbol{\Psi}_{1 i}(\xi, s) d \xi+\int_{x_{0}}^{x_{2}} \exp \left(\alpha\left(x_{0}-\xi\right)\right) \boldsymbol{\Psi}_{1 i}(\xi, s) d \xi, \quad i=1, \cdots, 6 \\
\bar{F}\left(x_{0}, s\right)=F\left(x_{0}, s\right)-s G(s)\left[\int_{x_{1}}^{x_{0}} \exp \left(\alpha\left(\xi-x_{0}\right)\right) \boldsymbol{\eta}_{q 1}(\xi, s) d \xi \quad\right. \\
\left.+\int_{x_{0}}^{x_{2}} \exp \left(\alpha\left(x_{0}-\xi\right)\right) \boldsymbol{\eta}_{q 1}(\xi, s) d \xi\right]
\end{gathered}
$$

and

$$
\begin{aligned}
\bar{F}^{I}\left(x_{0}, s\right)=F^{I}\left(x_{0}, s\right)+\alpha s G(s)\left[\int_{x_{1}}^{x_{0}} \exp \left(\alpha\left(\xi-x_{0}\right)\right) \boldsymbol{\eta}_{q 1}(\xi, s) d \xi\right. & \\
& \left.-\int_{x_{0}}^{x_{2}} \exp \left(\alpha\left(x_{0}-\xi\right)\right) \boldsymbol{\eta}_{q 1}(\xi, s) d \xi d \tau\right]
\end{aligned}
$$

The vector $\mathbf{v}_{0}(s)$ can be expressed in terms of vector $\mathbf{u}_{0}(s)$ as

$$
\mathbf{v}_{0}(s)=\left\{\begin{array}{c}
W^{I V}\left(x_{0}, s\right) \\
W^{V}\left(x_{0}, s\right)
\end{array}\right\}=\mathbf{Z}_{1}^{-1} \mathbf{Z}_{2} \mathbf{u}_{0}(s)+\mathbf{Z}_{1}^{-1}\left\{\begin{array}{c}
F\left(x_{0}, s\right) \\
F^{I}\left(x_{0}, s\right)
\end{array}\right\}=\mathbf{Z}_{1}^{-1} \mathbf{Z}_{2}\left\{\begin{array}{c}
W\left(x_{0}, s\right) \\
W^{I}\left(x_{0}, s\right) \\
W^{I I}\left(x_{0}, s\right) \\
W^{I I I}\left(x_{0}, s\right)
\end{array}\right\}+\mathbf{v}_{0 F}(s)
$$


where

$$
\begin{aligned}
& \mathbf{Z}_{1}=\left[\begin{array}{cc}
E I+s G(s) \boldsymbol{\Theta}_{15} & s G(s) \boldsymbol{\Theta}_{16} \\
\alpha s G(s) \boldsymbol{\Xi}_{15} & E I+\alpha s G(s) \boldsymbol{\Xi}_{16}
\end{array}\right] \\
& \mathbf{Z}_{2}=-\left[\begin{array}{cccc}
s G(s) \boldsymbol{\Theta}_{11}+s^{2} \rho A & s G(s) \boldsymbol{\Theta}_{12} & s G(s) \boldsymbol{\Theta}_{13} & s G(s) \boldsymbol{\Theta}_{14} \\
\alpha s G(s) \boldsymbol{\Xi}_{11} & \alpha s G(s) \boldsymbol{\Xi}_{12}+s^{2} \rho A & \alpha s G(s) \boldsymbol{\Xi}_{13} & \alpha s G(s) \boldsymbol{\Xi}_{14}
\end{array}\right] \\
& \text { and } \quad \mathbf{v}_{0 F}=\mathbf{Z}_{1}^{-1}\left\{\begin{array}{c}
\bar{F}\left(x_{0}, s\right) \\
\bar{F}^{I}\left(x_{0}, s\right)
\end{array}\right\}
\end{aligned}
$$

Substituting Eq. (40) into Eq. (27) we have

$$
\begin{aligned}
& \boldsymbol{\eta}(x, s)=\left\{\begin{array}{c}
\mathbf{u}(x, s) \\
\mathbf{v}(x, s)
\end{array}\right\}=\boldsymbol{\eta}_{q}(x, s)+\exp \left(\boldsymbol{\Phi}(s)\left(x-x_{0}\right)\right)\left(\left[\begin{array}{c}
\mathbf{I}_{4 \times 4} \\
\mathbf{Z}_{1}^{-1} \mathbf{Z}_{2}
\end{array}\right] \mathbf{u}_{0}(s)+\left\{\begin{array}{c}
\mathbf{0}_{4 \times 1} \\
\mathbf{v}_{0 F}(s)
\end{array}\right\}\right) \\
& x \in\left(x_{1}, x_{2}\right)
\end{aligned}
$$

For convenience partition

$$
\begin{aligned}
\boldsymbol{\Psi}(x, s) & =\exp \left(\boldsymbol{\Phi}(s)\left(x-x_{0}\right)\right)=\left[\begin{array}{ll}
\boldsymbol{\Psi}_{u u}(x, s) & \boldsymbol{\Psi}_{u v}(x, s) \\
\boldsymbol{\Psi}_{v u}(x, s) & \boldsymbol{\Psi}_{v v}(x, s)
\end{array}\right] \\
\text { and } \quad \boldsymbol{\eta}_{q}(x, s) & =\left\{\begin{array}{l}
\mathbf{u}_{q}(x, s) \\
\mathbf{v}_{q}(x, s)
\end{array}\right\},
\end{aligned}
$$

where $\boldsymbol{\Psi}_{u u}(x, s) \in \mathbb{C}^{4 \times 4}, \boldsymbol{\Psi}_{v v}(x, s) \in \mathbb{C}^{2 \times 2} \mathbf{u}_{q}(x, s) \in \mathbb{C}^{4 \times 1}$ and $\mathbf{v}_{q}(x, s) \in \mathbb{C}^{2 \times 1}$. From Eq. (44), we can obtain the solution of state vector $\mathbf{u}(x, s)$ as

$$
\mathbf{u}(x, s)=\left\{\begin{array}{c}
W(x, s) \\
W^{I}(x, s) \\
W^{I I}(x, s) \\
W^{I I I}(x, s)
\end{array}\right\}=\mathbf{u}_{q}(x, s)+\left[\mathbf{\Psi}_{u u}(x, s)+\mathbf{\Psi}_{u v}(x, s) \mathbf{Z}_{1}^{-1} \mathbf{Z}_{2}\right] \mathbf{u}_{0}(s)+\mathbf{\Psi}_{u v}(x, s) \mathbf{v}_{0 F}(s)
$$

where $\mathbf{u}_{q}(x, s)$ and $\mathbf{v}_{0 F}(s)$ can be determined from the external load $f(x, t)$. Eq. (47) is therefore the solution of Eq. (16). For the free vibration $f(x, t)=0$, and consequently from the above equations $\mathbf{u}_{q}(x, s)=0$ and $\mathbf{v}_{0 F}(s)=0$. Defining a transfer matrix

$$
\mathbf{T}(x, s)=\mathbf{\Psi}_{u u}(x, s)+\mathbf{\Psi}_{u v}(x, s) \mathbf{Z}_{1}^{-1} \mathbf{Z}_{2}
$$

Eq. (47) becomes

$$
\mathbf{u}(x, s)=\mathbf{T}(x, s) \mathbf{u}_{0}(s),
$$

\section{Transfer Matrix Solution for the Uniformly Viscously Damped Segments}

Applying the Laplace transform to the equation of motion for the uniformly viscously damped segments (15), and considering the state-vector $\mathbf{u}(x, s)$ we can obtain

$$
\frac{d}{d x} \mathbf{u}(x, s)=\overline{\mathbf{\Phi}}(s) \mathbf{u}(x, s)+\overline{\mathbf{q}}(x, s), \quad x \in\left(x_{L}, x_{1}\right) \cup\left(x_{2}, x_{R}\right)
$$

where

$$
\overline{\mathbf{\Phi}}(s)=\left[\begin{array}{cccc}
0 & 1 & 0 & 0 \\
0 & 0 & 1 & 0 \\
0 & 0 & 0 & 1 \\
-\frac{s^{2} \rho A+s C_{0}}{E I} & 0 & 0 & 0
\end{array}\right] \text { and } \quad \overline{\mathbf{q}}(x, s)=\left\{\begin{array}{c}
0 \\
0 \\
0 \\
\frac{F(x, s)}{E I}
\end{array}\right\}
$$

$$
8 \text { of } 16
$$


Eq. (50) can be solved following the procedure outlined earlier to give

$$
\mathbf{u}(x, s)=\int_{x_{1}}^{x} \exp (\overline{\mathbf{\Phi}}(s)(x-\xi)) \mathbf{q}(\xi, s) d \xi+\exp \left(\overline{\mathbf{\Phi}}(s)\left(x-x_{1}\right)\right) \mathbf{u}\left(x_{1}, s\right)
$$$$
\text { when } x \in\left(x_{L}, x_{1}\right)
$$

and

$$
\mathbf{u}(x, s)=\int_{x_{2}}^{x} \exp (\overline{\mathbf{\Phi}}(s)(x-\xi)) \mathbf{q}(\xi, s) d \xi+\exp \left(\overline{\mathbf{\Phi}}(s)\left(x-x_{2}\right)\right) \mathbf{u}\left(x_{2}, s\right) \quad \text { when } x \in\left(x_{2}, x_{R}\right)
$$

\section{Transfer Matrix Solution for the Complete Beam} have

For free vibration $\overline{\mathbf{q}}(x, s)=\mathbf{0}$. Considering $x=x_{L}$ and $x=x_{R}$ in Eqs. (52) and (53) respectively we

$$
\begin{aligned}
\mathbf{u}\left(x_{L}, s\right) & =\exp \left(\overline{\mathbf{\Phi}}(s)\left(x_{L}-x_{1}\right)\right) \mathbf{u}\left(x_{1}, s\right) \\
\text { and } \quad \mathbf{u}\left(x_{R}, s\right) & =\exp \left(\overline{\mathbf{\Phi}}(s)\left(x_{R}-x_{2}\right)\right) \mathbf{u}\left(x_{2}, s\right)
\end{aligned}
$$

Using Eq. (49) for $x=x_{1}$ and $x=x_{2}$ and substituting into Eqs. (54) and (55) respectively one obtains

$$
\begin{aligned}
\mathbf{u}\left(x_{L}, s\right) & =\exp \left(\overline{\mathbf{\Phi}}(s)\left(x_{L}-x_{1}\right)\right) \mathbf{T}\left(x_{1}, s\right) \mathbf{u}_{0}(s) \\
\text { and } \quad \mathbf{u}\left(x_{R}, s\right) & =\exp \left(\overline{\mathbf{\Phi}}(s)\left(x_{R}-x_{2}\right)\right) \mathbf{T}\left(x_{2}, s\right) \mathbf{u}_{0}(s)
\end{aligned}
$$

The boundary conditions of beam can be expressed as

$$
\mathbf{M}(s) \mathbf{u}\left(x_{L}, s\right)+\mathbf{N}(s) \mathbf{u}\left(x_{R}, s\right)=\mathbf{0}
$$

where $\mathbf{M} \in \mathbb{C}^{4 \times 4}$ and $\mathbf{N} \in \mathbb{C}^{4 \times 4}$ are the boundary matrices. Boundary matrices arising due to different type of boundary conditions are discussed in section VII. Substituting Eqs. (56) and (57) into Eq. (58) one obtains

$$
\left[\mathbf{M}(s) \exp \left(\overline{\mathbf{\Phi}}(s)\left(x_{L}-x_{1}\right)\right) \mathbf{T}\left(x_{1}, s\right)+\mathbf{N}(s) \exp \left(\overline{\mathbf{\Phi}}(s)\left(x_{R}-x_{2}\right)\right) \mathbf{T}\left(x_{2}, s\right)\right] \mathbf{u}_{0}(s)=\mathbf{0}
$$

The eigenvalues are the roots of characteristic equation

$$
\operatorname{det}\left[\mathbf{M}(s) \exp \left(\overline{\mathbf{\Phi}}(s)\left(x_{L}-x_{1}\right)\right) \mathbf{T}\left(x_{1}, s\right)+\mathbf{N}(s) \exp \left(\overline{\mathbf{\Phi}}(s)\left(x_{R}-x_{2}\right)\right) \mathbf{T}\left(x_{2}, s\right)\right]=0
$$

Assuming $\lambda_{j}$ are the eigenvalues, the corresponding mode shapes can be determined by

$$
\boldsymbol{\psi}_{j}(x)=\mathbf{u}\left(x, \lambda_{j}\right)=\left\{\begin{array}{l}
\exp \left(\overline{\mathbf{\Phi}}\left(\lambda_{j}\right)\left(x-x_{1}\right)\right) \mathbf{T}\left(x_{1}, \lambda_{j}\right) \mathbf{u}_{0}\left(\lambda_{j}\right), \quad x_{L} \leq x \leq x_{1} \\
\mathbf{T}\left(x, \lambda_{j}\right) \mathbf{u}_{0}\left(\lambda_{j}\right), \quad x_{1} \leq x \leq x_{2} \\
\exp \left(\overline{\mathbf{\Phi}}\left(\lambda_{j}\right)\left(x-x_{2}\right)\right) \mathbf{T}\left(x_{2}, \lambda_{j}\right) \mathbf{u}_{0}\left(\lambda_{j}\right), \quad x_{2} \leq x \leq x_{R}
\end{array}\right.
$$

\section{Incorporation of Different Boundary Conditions}

In this section we discuss how different types of boundary conditions can be incorporated within the formulation presented so far. Note that the boundary conditions only affect the matrices $\mathbf{M}(s)$ and $\mathbf{N}(s)$ introduced in Eq. (58). First the following simple cases are considered:

- Clamped-clamped (C-C) boundary condition: In this case we have

$$
w(x, t)=0 \quad \text { and } \quad \frac{\partial}{\partial x} w(x, t)=0 \quad \text { for } \quad x=x_{L} \quad \text { and } \quad x=x_{R}
$$

Taking the Laplace transform and considering the definition of $\mathbf{u}$ in Eq. (23) we have

$$
\mathbf{M}(s)=\left[\begin{array}{ll}
\mathbf{I}_{2 \times 2} & \mathbf{O}_{2 \times 2} \\
\mathbf{O}_{2 \times 2} & \mathbf{O}_{2 \times 2}
\end{array}\right] \text { and } \mathbf{N}(s)=\left[\begin{array}{cc}
\mathbf{O}_{2 \times 2} & \mathbf{O}_{2 \times 2} \\
\mathbf{I}_{2 \times 2} & \mathbf{O}_{2 \times 2}
\end{array}\right]
$$

where $\mathbf{I}_{2 \times 2}$ and $\mathbf{O}_{2 \times 2}$ are the $2 \times 2$ identity and null matrices respectively.

$$
9 \text { of } 16
$$


- Free-Free (F-F) boundary condition: In this case we have

$$
\frac{\partial^{2}}{\partial x^{2}} w(x, t)=0 \quad \text { and } \quad \frac{\partial^{3}}{\partial x^{3}} w(x, t)=0 \quad \text { for } \quad x=x_{L} \quad \text { and } \quad x=x_{R}
$$

Taking the Laplace transform and considering the definition of $\mathbf{u}$ in Eq. (23) we have

$$
\mathbf{M}(s)=\left[\begin{array}{ll}
\mathbf{O}_{2 \times 2} & \mathbf{I}_{2 \times 2} \\
\mathbf{O}_{2 \times 2} & \mathbf{O}_{2 \times 2}
\end{array}\right] \text { and } \mathbf{N}(s)=\left[\begin{array}{cc}
\mathbf{O}_{2 \times 2} & \mathbf{O}_{2 \times 2} \\
\mathbf{O}_{2 \times 2} & \mathbf{I}_{2 \times 2}
\end{array}\right]
$$

- Pinned-Pinned (P-P) boundary condition: In this case we have

$$
w(x, t)=0 \quad \text { and } \quad \frac{\partial^{2}}{\partial x^{2}} w(x, t)=0 \quad \text { for } \quad x=x_{L} \quad \text { and } \quad x=x_{R}
$$

Taking the Laplace transform and considering the definition of $\mathbf{u}$ in Eq. (23) we have

$$
\mathbf{M}(s)=\left[\begin{array}{llll}
1 & 0 & 0 & 0 \\
0 & 0 & 1 & 0 \\
0 & 0 & 0 & 0 \\
0 & 0 & 0 & 0
\end{array}\right] \text { and } \mathbf{N}(s)=\left[\begin{array}{llll}
0 & 0 & 0 & 0 \\
0 & 0 & 0 & 0 \\
1 & 0 & 0 & 0 \\
0 & 0 & 1 & 0
\end{array}\right]
$$

Following a similar approach, the boundary matrices for other type of simple boundary conditions, for example clamped-pinned (C-P) or clamped-free (C-F), can also be derived.

For more complex boundary conditions, such as general elastic restraints and/or lumped damping, the boundary matrices $\mathbf{M}(s)$ and $\mathbf{N}(s)$ can also be obtained easily. As an example we consider an EulerBernoulli beam with complex boundary conditions shown in figure 2 . The boundary conditions at the right

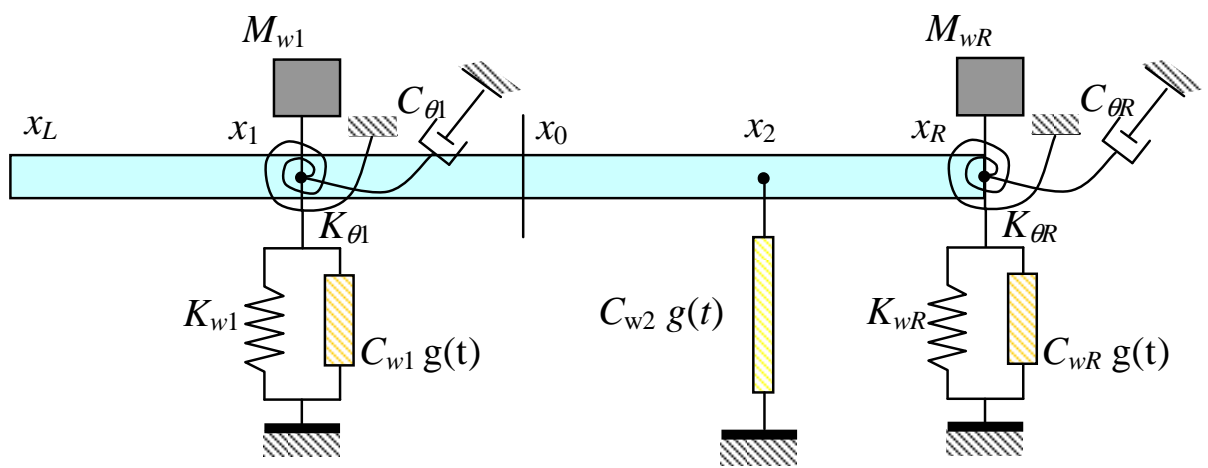

Figure 2. Euler-Bernoulli beam with complex boundary conditions and middle supports.

end $\left(x=x_{R}\right)$ are given by

$$
\begin{array}{r}
M_{w R} \frac{\partial^{2} w}{\partial t^{2}}\left(x_{R}, t\right)+\left.\int_{-\infty}^{t} C_{w R} \mu \exp (-\mu(t-\tau)) \frac{\partial w\left(x_{R}, t\right)}{\partial t}\right|_{t=\tau} d \tau+K_{w R} w\left(x_{R}, t\right)=\left.E I \frac{\partial^{3} w(x, t)}{\partial x^{3}}\right|_{x=x_{R}} \\
\left.C_{\theta R} \frac{\partial^{2} w(x, t)}{\partial x \partial t}\right|_{x=x_{R}}+\left.K_{\theta R} \frac{\partial w(x, t)}{\partial x}\right|_{x=x_{R}}=-\left.E I \frac{\partial^{2} w(x, t)}{\partial x^{2}}\right|_{x=x_{R}}
\end{array}
$$

Laplace transforms of Eqs. (68) and (69) lead to

$$
\begin{array}{r}
-\left(M_{w R} s^{2}+C_{w R} \frac{\mu s}{\mu+s}+K_{w R}\right) W\left(x_{R}, s\right)+\left.E I \frac{\partial^{3} W(x, s)}{\partial x^{3}}\right|_{x=x_{R}}=0 \\
\left.\left(C_{\theta R} s+K_{\theta R}\right) \frac{\partial W(x, s)}{\partial x}\right|_{x=x_{R}}+\left.E I \frac{\partial^{2} W(x, s)}{\partial x^{2}}\right|_{x=x_{R}}=0
\end{array}
$$

$$
10 \text { of } 16
$$


Using Eqs. (70) and (71) the matrix $\mathbf{N}(s)$ can be obtained as

$$
\mathbf{N}(s)=\left[\begin{array}{cccc}
0 & 0 & 0 & 0 \\
0 & 0 & 0 & 0 \\
0 & \frac{1}{E I}\left(C_{\theta R} s+K_{\theta R}\right) & 1 & 0 \\
-\frac{1}{E I}\left(M_{w R} s^{2}+C_{w R} \frac{\mu s}{s+\mu}+K_{w R}\right) & 0 & 0 & 1
\end{array}\right]
$$

In figure 2 the supports at points $x_{1}$ and $x_{2}$ influence the third and forth elements of the state vector $\mathbf{u}(x, s)$, which are related to the bending moment and shear force. This can be taken into account by introducing two state transformation matrices $\mathbf{B}_{1}(s)$ and $\mathbf{B}_{2}(s)$. Equations (56) and (57) are modified to give

$$
\begin{aligned}
\mathbf{u}\left(x_{L}, s\right) & =\exp \left(\overline{\mathbf{\Phi}}(s)\left(x_{L}-x_{1}\right)\right) \mathbf{B}_{1}^{-1}(s) \mathbf{T}\left(x_{1}, s\right) \mathbf{u}_{0}(s) \\
\text { and } \quad \mathbf{u}\left(x_{R}, s\right) & =\exp \left(\overline{\mathbf{\Phi}}(s)\left(x_{R}-x_{2}\right)\right) \mathbf{B}_{2}(s) \mathbf{T}\left(x_{2}, s\right) \mathbf{u}_{0}(s)
\end{aligned}
$$

where

$$
\begin{aligned}
\mathbf{B}_{1}(s) & =\left[\begin{array}{cccc}
1 & 0 & 0 & 0 \\
0 & 1 & 0 & 0 \\
0 & \frac{1}{E I}\left(K_{\theta 1}+C_{\theta 1} s\right) & 1 & 0 \\
-\frac{1}{E I}\left(K_{w 1}+M_{w 1} s^{2}+C_{w 1} \frac{\mu s}{s+\mu}\right) & 0 & 0 & 1
\end{array}\right] \\
\text { and } \mathbf{B}_{2}(s) & =\left[\begin{array}{cccc}
1 & 0 & 0 & 0 \\
0 & 1 & 0 & 0 \\
0 & 0 & 1 & 0 \\
-\frac{C_{w 2}}{E I} \frac{\mu s}{s+\mu} & 0 & 0 & 1
\end{array}\right]
\end{aligned}
$$

These two matrices are obtained using the displacement continuity and force balance relationships at the support points $x_{1}$ and $x_{2}$ respectively.

\section{Numerical Examples}

\section{A. Example 1: Damped Beam with Step Variation in the System Properties}

The beam example considered here is taken from reference ${ }^{11}$ and shown in figure 3 . The purpose of this

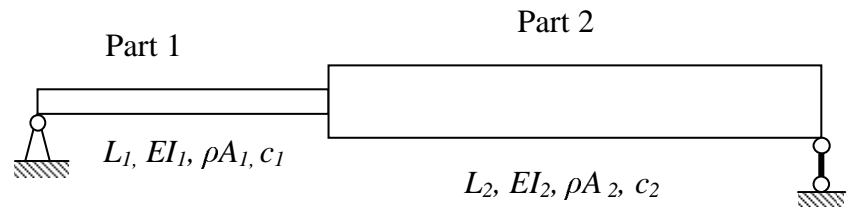

Figure 3. Damped beam with step variation in the system properties with pinned-pinned boundary conditions.

example is to compare the results obtained from the proposed theory for a special case against published literature. The numerical values of the physical properties for the two parts are shown in table 1. The eigenvalues of the beam are obtained by solving Eq. (60) and the results are shown in table 2 
Table 1. Numerical values of the physical properties of the beam.

\begin{tabular}{cll} 
Parameters & Part 1 & Part 2 \\
\hline$L_{i}$ & $1 \mathrm{~m}$ & $2 \mathrm{~m}$ \\
$\rho A_{i}$ & $10 \mathrm{~kg} / \mathrm{m}$ & $20 \mathrm{~kg} / \mathrm{m}$ \\
$c_{i}$ & $0 \mathrm{Ns} / \mathrm{m}^{2}$ & $10 \mathrm{Ns} / \mathrm{m}^{2}$ \\
$E I_{i}$ & $100 \mathrm{Nm}^{2}$ & $100 \mathrm{Nm}^{2}$
\end{tabular}

Table 2. The first eight eigenvalues of the beam.

\begin{tabular}{cc} 
Proposed method & Reference $^{11}$ \\
\hline$-2.2552 \pm 1.2711 \mathrm{i}$ & $-2.2552 \pm 1.2711 \mathrm{i}$ \\
$-1.7936 \pm 10.903 \mathrm{i}$ & $-1.7936 \pm 10.903 \mathrm{i}$ \\
$-1.5741 \pm 24.863 \mathrm{i}$ & $-1.5741 \pm 24.863 \mathrm{i}$ \\
$-1.7876 \pm 43.165 \mathrm{i}$ & $-1.7876 \pm 43.165 \mathrm{i}$ \\
$-1.8781 \pm 68.118 \mathrm{i}$ & $-1.8781 \pm 68.118 \mathrm{i}$ \\
$-1.6984 \pm 99.327 \mathrm{i}$ & $-1.6984 \pm 99.327 \mathrm{i}$ \\
$-1.6775 \pm 133.66 \mathrm{i}$ & $-1.6775 \pm 133.66 \mathrm{i}$ \\
$-1.8549 \pm 174.00 \mathrm{i}$ & $-1.8549 \pm 174.00 \mathrm{i}$
\end{tabular}

\section{B. Example 2: Damped Beam with Complex Boundary Conditions}

A damped beam with complex boundary conditions and middle supports is considered. Figure 4 shows the beam in its most general configuration. Two special cases for the damping models are considered. For the

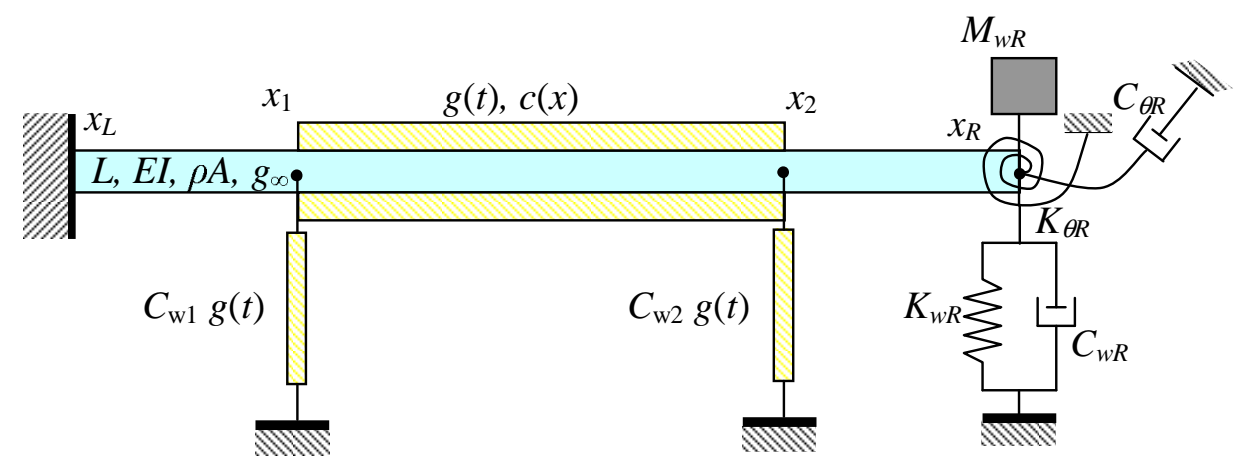

Figure 4. Euler-Bernoulli beam with complex boundary conditions and middle supports.

first case the damping is assumed to be standard non-local viscous and the parameter values are taken from reference. ${ }^{12}$ For the second case it is assumed that the damping is non-local and non-viscous. The numerical values (in SI units) of the system parameters for the two cases are as follows:

- Case 1: Local viscous damping:

$L=1, E I=1, m=16, M_{w R}=4, K_{w R}=8, C_{w R}=4, g_{\infty}=1.6, g(t)=\delta(t), \mathrm{c}(x)=\delta(x)$,

$C_{\theta R}=K_{\theta R}=C_{w 1}=C_{w 2}=0$

- Case 2: Non-local non-viscous damping:

$L=1, E I=1, m=16, M_{w R}=4, K_{w R}=8, C_{w R}=4, g_{\infty}=16, g(t)=\mu \exp (-\mu t)$, 


$$
c(x)=\alpha \exp (-\alpha|x|), K_{\theta R}=8, C_{\theta R}=C_{w 1}=C_{w 2}=4
$$

Table 3 compares the first ten eigenvalues for case 1, computed using the proposed method, with those obtained in reference. ${ }^{12}$ Clearly the proposed method produces identical results.

Table 3. The first ten eigenvalues of the beam for case 1 .

\begin{tabular}{ccc}
$j$ & \multicolumn{2}{c}{$\lambda_{j}$} \\
\cline { 2 - 3 } & Proposed method & Reference $^{12}$ \\
\hline 1 & $-0.2705 \pm 1.1451 \mathrm{i}$ & $-0.2705+1.1451 \mathrm{i}$ \\
2 & $-0.1357 \pm 4.4930 \mathrm{i}$ & $-0.1357+4.4930 \mathrm{i}$ \\
3 & $-0.0896 \pm 13.2586 \mathrm{i}$ & $-0.0896+13.2586 \mathrm{i}$ \\
4 & $-0.0727 \pm 26.8877 \mathrm{i}$ & $-0.0727+26.8877 \mathrm{i}$ \\
5 & $-0.0647 \pm 45.4297 \mathrm{i}$ & $-0.0647+45.4297 \mathrm{i}$ \\
6 & $-0.0602 \pm 68.8941 \mathrm{i}$ & $-0.0602+68.8941 \mathrm{i}$ \\
7 & $-0.0575 \pm 97.2862 \mathrm{i}$ & $-0.0575+97.2862 \mathrm{i}$ \\
8 & $-0.0557 \pm 130.6088 \mathrm{i}$ & $-0.0557+130.6088 \mathrm{i}$ \\
9 & $-0.0546 \pm 168.8632 \mathrm{i}$ & $-0.0546+168.8632 \mathrm{i}$ \\
10 & $-0.0537 \pm 212.0505 \mathrm{i}$ & $-0.0537+212.0505 \mathrm{i}$
\end{tabular}

Table 4 shown the first ten eigenvalues for case 2 for different values of relaxation parameters. The real

Table 4. The first ten eigenvalues of the beam for case 2 .

\begin{tabular}{cccccc}
\hline \multirow{5}{*}{$j$} & \multicolumn{4}{c}{$\lambda_{j}$} \\
\cline { 2 - 5 } & $\mu=\infty, \quad \alpha=\infty$ & $\mu=100, \quad \alpha=10$ & $\mu=100, \alpha=0.1$ & $\mu=1, \quad \alpha=10$ \\
\hline 1 & $-0.67921 \pm 1.1780 \mathrm{i}$ & $-0.65439 \pm 1.1966 \mathrm{i}$ & $-0.56874 \pm 1.2444 \mathrm{i}$ & $-0.52895 \pm 1.4404 \mathrm{i}$ \\
2 & $-0.98559 \pm 6.4492 \mathrm{i}$ & $-0.92155 \pm 6.4835 \mathrm{i}$ & $-0.61797 \pm 6.4677 \mathrm{i}$ & $-0.49096 \pm 6.5182 \mathrm{i}$ \\
3 & $-1.3833 \pm 16.611 \mathrm{i}$ & $-1.2245 \pm 16.723 \mathrm{i}$ & $-1.0592 \pm 16.703 \mathrm{i}$ & $-0.61363 \pm 16.656 \mathrm{i}$ \\
4 & $-1.4263 \pm 31.584 \mathrm{i}$ & $-1.2455 \pm 31.754 \mathrm{i}$ & $-1.1706 \pm 31.727 \mathrm{i}$ & $-0.73244 \pm 31.620 \mathrm{i}$ \\
5 & $-1.0714 \pm 51.442 \mathrm{i}$ & $-0.90384 \pm 51.521 \mathrm{i}$ & $-0.83720 \pm 51.483 \mathrm{i}$ & $-0.74997 \pm 51.456 \mathrm{i}$ \\
6 & $-1.0353 \pm 76.208 \mathrm{i}$ & $-0.79867 \pm 76.276 \mathrm{i}$ & $-0.74770 \pm 76.237 \mathrm{i}$ & $-0.71290 \pm 76.207 \mathrm{i}$ \\
7 & $-1.3874 \pm 105.87 \mathrm{i}$ & $-0.92919 \pm 106.11 \mathrm{i}$ & $-0.90512 \pm 106.08 \mathrm{i}$ & $-0.71677 \pm 105.88 \mathrm{i}$ \\
8 & $-1.4200 \pm 140.46 \mathrm{i}$ & $-0.91144 \pm 140.68 \mathrm{i}$ & $-0.89997 \pm 140.67 \mathrm{i}$ & $-0.75157 \pm 140.47 \mathrm{i}$ \\
9 & $-1.0745 \pm 179.97 \mathrm{i}$ & $-0.78527 \pm 180.03 \mathrm{i}$ & $-0.77680 \pm 180.01 \mathrm{i}$ & $-0.75400 \pm 179.98 \mathrm{i}$ \\
10 & $-1.0541 \pm 224.42 \mathrm{i}$ & $-0.74574 \pm 224.45 \mathrm{i}$ & $-0.74022 \pm 224.44 \mathrm{i}$ & $-0.73084 \pm 224.42 \mathrm{i}$
\end{tabular}

parts of the first four modes are shown in figure 5 for the four sets of parameter values given in table 4. Note that $\mu$ and $\alpha$ do not affect the real part of modes significantly. The imaginary parts of the first four modes are shown in figure 6 . Note that $\mu$ and $\alpha$ do significantly affect the imaginary parts of modes.

\section{Conclusions}

The increasing use of advanced composite materials and active control mechanisms demand sophisticated treatment of damping forces within a distributed parameter system. This paper proposes a method to obtain the natural frequencies and mode-shapes of Euler-Bernoulli beams with general linear damping models. It was assumed that the damping force at a given point in the beam depends on the past history of velocities at different points via convolution integrals over exponentially decaying kernel functions. Conventional viscous and viscoelastic damping models can be obtained as special cases of this general linear damping model. The proposed method was applied to two example problems. For both problems, the results obtained from the proposed method for some special cases are compared against published results. Good agreements were found for the test problems. Future work will discuss computational issues and forced vibration problems. 


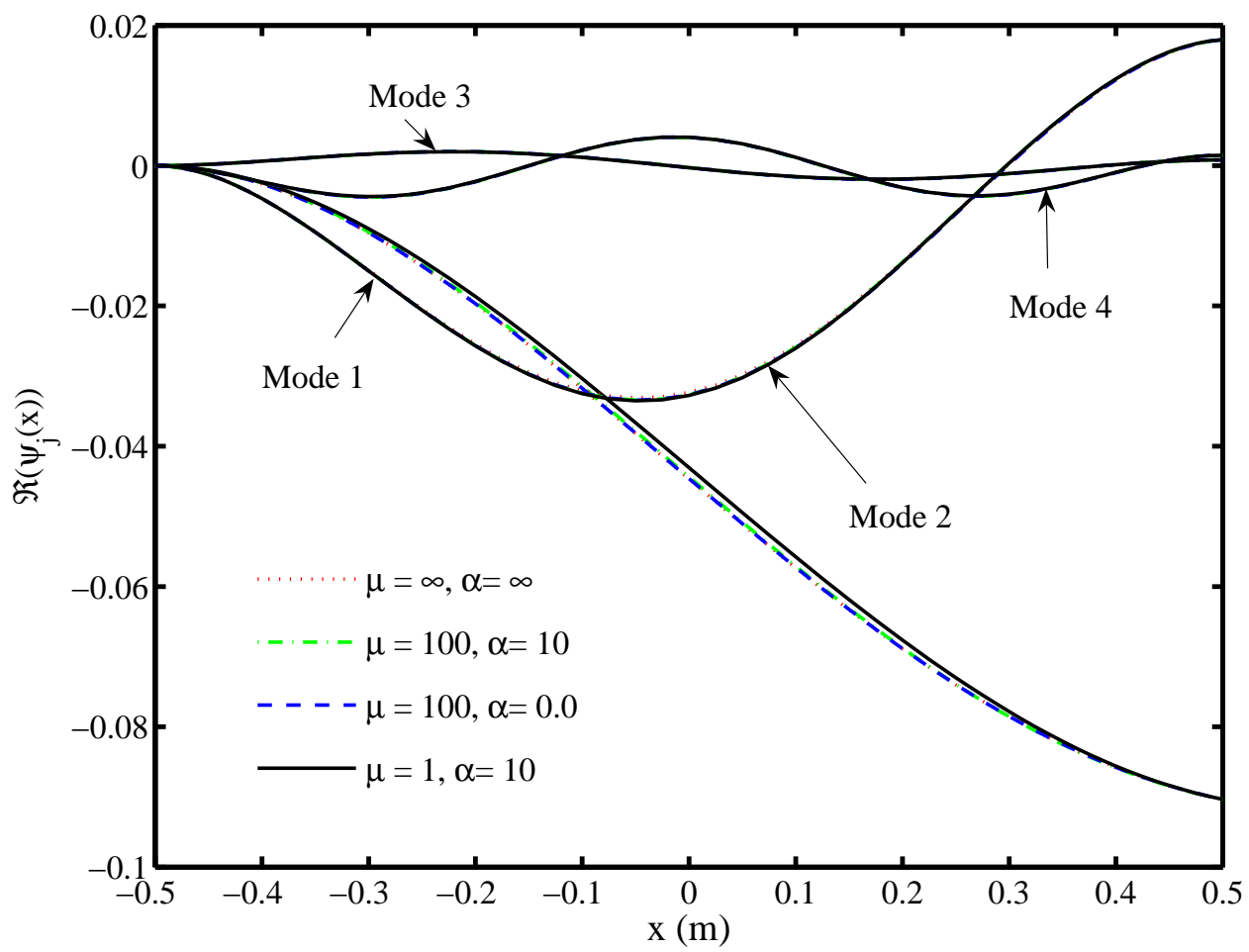

Figure 5. The real parts of the first four modes for case 2 .

\section{Acknowledgements}

Sondipon Adhikari gratefully acknowledges the support of the Engineering and Physical Sciences Research Council through the award of an Advanced Research Fellowship. Y. Lei gratefully acknowledges the support of China Scholarship Council through a Scholarship Fund Award. Michael Friswell gratefully acknowledges the support of the Royal Society through a Royal Society-Wolfson Research Merit Award.

\section{References}

${ }^{1}$ Lord Rayleigh, Theory of Sound (two volumes), Dover Publications, New York, 1945th ed., 1877.

${ }^{2}$ Lazan, B. J., Damping of Materials and Members in Structural Mechanics, Pergamon Press, Oxford, 1968.

${ }^{3}$ Bert, C. W., "Material damping: an introductory review of mathematical models, measure and experimental techniques," Journal of Sound and Vibration, Vol. 29, No. 2, 1973, pp. 129-153.

${ }^{4}$ Ungar, E. E., "The status of engineering knowledge concerning the damping of built-up structures," Journal of Sound and Vibration, Vol. 26, 1973, pp. 141-154.

${ }^{5}$ Nashif, A. D., Jones, D. I. G., and Henderson, J. P., Vibration Damping, John Wiley, New York, 1985.

${ }^{6}$ Adhikari, S., "Dynamics of non-viscously damped linear systems," ASCE Journal of Engineering Mechanics, Vol. 128, No. 3, March 2002, pp. 328-339.

${ }^{7}$ Wagner, N. and Adhikari, S., "Symmetric state-space formulation for a class of non-viscously damped systems," AIAA Journal, Vol. 41, No. 5, 2003, pp. 951-956.

${ }^{8}$ Banks, H. T. and Inman, D. J., "On damping mechanisms in beams," Transactions of ASME, Journal of Applied Mechanics, Vol. 58, September 1991, pp. 716-723.

${ }^{9}$ Caughey, T. K. and O'Kelly, M. E. J., "Classical normal modes in damped linear dynamic systems," Transactions of ASME, Journal of Applied Mechanics, Vol. 32, September 1965, pp. 583-588.

${ }^{10}$ Yang, B. and Tan, C. A., "Transfer functions of one-dimensional distributed parameter systems," Transactions of ASME, Journal of Applied Mechanics, Vol. 59, No. 4, 1992, pp. 1009-1014.

${ }^{11}$ Friswell, M. I. and Lees, A. W., "The modes of non-homogeneous damped beams," Journal of Sound and Vibration, Vol. 242, No. 2, 2001, pp. 355-361.

${ }^{12}$ Yang, B. and Wu, X., "Transient response of one- dimensional distributed systems: a closed form eignfunction expansion realization," Journal of Sound and Vibration, Vol. 208, No. 5, 1997, pp. 763-776. 

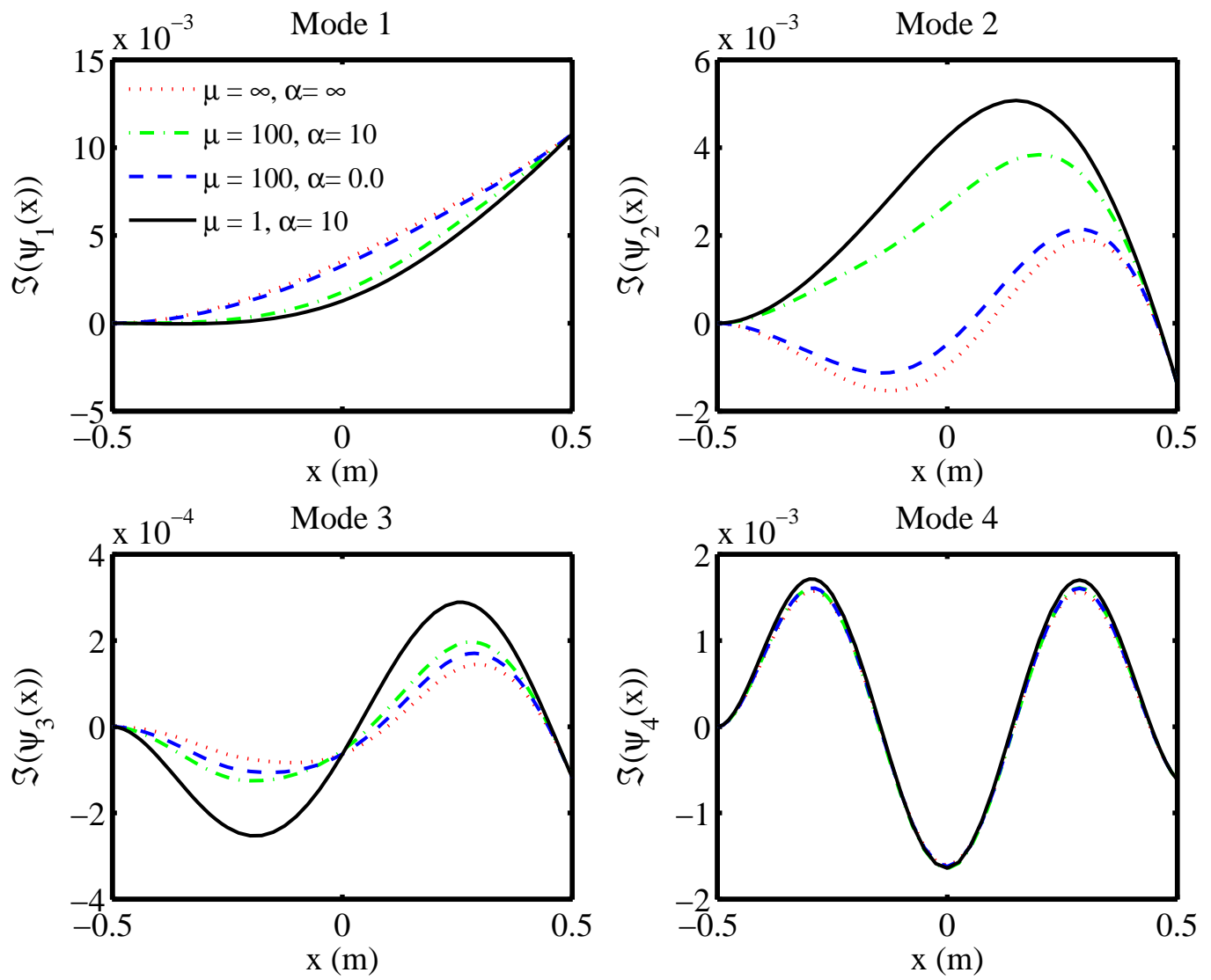

Figure 6. The imaginary parts of the first four modes for case 2.

\section{Nomenclature}

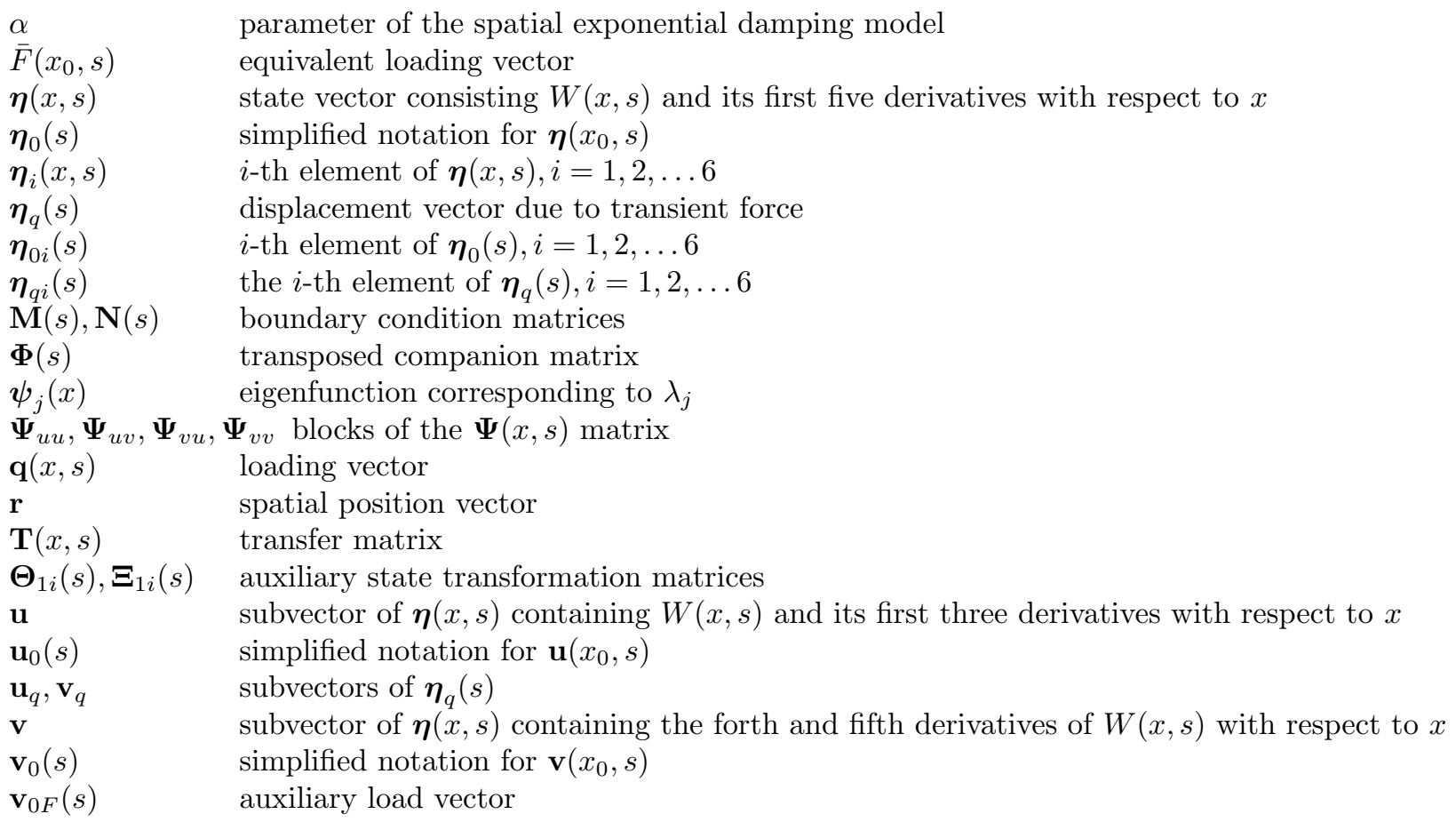




\begin{tabular}{|c|c|}
\hline $\mathbf{Z}_{1}(s), \mathbf{Z}_{2}(s)$ & transformation matrices \\
\hline $\mathcal{L}_{1}, \mathcal{L}_{2}$ & stiffness and damping operators \\
\hline $\mathcal{D}$ & spatial domain \\
\hline$(\ddot{\bullet})$ & second derivative of $(\bullet)$ with respect to $t$ \\
\hline$\delta(t)$ & Dirac-delta function \\
\hline $\mathcal{F}(t)$ & rate of energy dissipation \\
\hline$\Gamma_{i}$ & boundary surfaces \\
\hline$\hat{\mathcal{F}}(\omega)$ & dissipation energy in the frequency domain \\
\hline$\lambda_{j}$ & $j$-th eigenvalue of the beam \\
\hline$(\bullet)^{*}$ & complex conjugation of $(\bullet)$ \\
\hline $\mathcal{M}_{i}$ & boundary operators \\
\hline$\mu$ & parameter for the exponential damping model \\
\hline$\omega$ & angular frequency \\
\hline$\rho$ & line mass density distribution \\
\hline$\tau$ & dummy time variable \\
\hline$\xi$ & dummy spatial variable \\
\hline$A$ & section area \\
\hline$C(\bullet)$ & spatial damping distribution function \\
\hline$C_{0}$ & damping constant \\
\hline$C_{1}$ & general damping kernel function \\
\hline$E$ & Young's modulus \\
\hline$F(x, s)$ & Laplace transform of $f(x, t)$ \\
\hline$G(\omega)$ & Fourier transform of $g(t)$ \\
\hline$g(t)$ & damping function in the time domain \\
\hline$g_{\infty}$ & non-viscous damping coefficient \\
\hline$I$ & area moment of inertia \\
\hline$p(\mathbf{r}, t)$ & force distribution \\
\hline$s$ & Laplace domain parameter \\
\hline$t$ & time \\
\hline$u$ & displacement variable \\
\hline$U(\bullet, \omega)$ & Fourier transform of $u(\bullet, t)$ \\
\hline$W(x, s)$ & Laplace transform of $w(x, t)$ \\
\hline$w(x, t)$ & transverse displacement of the beam \\
\hline$x$ & spatial variable \\
\hline$x_{1}, x_{2}$ & boundary points of nonlocal damping patch \\
\hline$x_{L}, x_{R}$ & coordinate of the left and right end of the beam \\
\hline$\overline{\mathbf{q}}(x, s)$ & loading vector on the undamped beam segment \\
\hline$\overline{\mathbf{\Phi}}(s)$ & transposed companion matrix of the undamped beam segment \\
\hline $\mathrm{i}$ & unit imaginary number, $\mathrm{i}=\sqrt{-1}$ \\
\hline
\end{tabular}

Subscripts

$(\bullet)_{\theta}$

$(\bullet)_{w}$

Conventions $(\bullet)^{-1} \quad$ matrix inverse

$\Re(\bullet)$

Superscripts $(\bullet){ }^{I \cdots V I}$ quantity corresponding to the rotational degree of freedom quantity corresponding to the translational degree of freedom

first to sixth derivative with respect to $x$ 